\title{
A Tight Upper Bound on Channel Capacity for Visible Light Communications
}

\author{
Rui Jiang, Zhaocheng Wang, Qi Wang, and Linglong Dai
}

\begin{abstract}
Since the optical wireless channel in visible light communication (VLC) systems is subject to the nonnegativity of the signal and the average optical power, the classic Shannon channel capacity formula is not applicable to VLC systems. To derive a simple closed-form upper bound on channel capacity, sphere packing argument method has been applied previously. However, there is an obvious gap between the existing spherepacking upper bounds and the lower bounds at high optical signal-to-noise-ratios (OSNRs), which is mainly caused by the inaccurate mathematical approximation of the intrinsic volumes of the simplex. In this letter, a tight sphere-packing upper bound is derived with a new approximation method. Numerical results demonstrate that compared to the existing sphere-packing upper bounds, our proposed upper bound is tighter at high OSNRs.
\end{abstract}

Index Terms-Visible light communication, optical wireless channel, channel capacity, upper bound, sphere packing argument.

\section{INTRODUCTION}

Visible light communication (VLC), as a complementary technology to the conventional radio frequency communication, has drawn great attention recently due to its distinctive features such as unregulated spectrum, vast bandwidth, low electromagnetic interference and so on [1]. In VLC systems, shot noise and thermal noise at the receiver are often modeled as the additive white Gaussian noise (AWGN) [2], [3]. However, the capacity of the optical wireless channel is still an open issue. Although most literatures relevant to the channel capacity for VLC systems use the classic Shannon channel capacity formula [4], [5], it is not suitable for actual optical wireless channel due to the following reasons:

1) Intensity modulation with direct detection (IM/DD) is employed in VLC systems [6]. The intensity/amplitude of the signal would determine the voltage loaded on the light emitting diodes (LEDs) and the instantaneous radiated optical power. Thus, the signal is constrained to be nonnegative and realvalued.

2) Since LEDs are primarily used for illumination, the signal is subject to the average optical power instead of the electrical power. Therefore, the input signal distribution

This work was supported in part by the National Key Basic Research Program of China under Grant 2013CB329203, in part by the National Nature Science Foundation of China under Grant 61271266, in part by the Shenzhen Visible Light Communication System Key Laboratory under Grant ZDSYS20140512114229398, in part by the Shenzhen Wireless over Visible Light Communication Technology Engineering Laboratory under Grant SDRC[2012]No.1440, and in part by the Shenzhen Peacock Plan under Grant 1108170036003286 .

The authors are with the Tsinghua National Laboratory for Information Science and Technology, Department of Electronic Engineering, Tsinghua University, Beijing 100084, China (e-mail: jr14@mails.tsinghua.edu.cn; zcwang@tsinghua.edu.cn; qiwang11@mails.tsinghua.edu.cn; dail$1 @$ tsinghua.edu.cn). to approach channel capacity does not necessarily follow Gaussian distribution.

The capacity of the optical wireless channel with Gaussian noise has been investigated in recent years. Based on the wellknown sphere packing method [7], tight closed-form upper bounds are derived in [8], [9]. However, there is an obvious gap between these upper bounds and the lower bound in high optical signal-to-noise ratios (OSNRs) region. A recent independent work [10] produced an even tighter upper bound that is also based on the sphere-packing bound. In [3], another tight upper bound is derived using a dual expression for channel capacity. When only an average power constraint is imposed, it coincides with the lower bound at high OSNRs.

In this letter, a new mathematical approximation method for the intrinsic volumes of the simplex is proposed to derive a tight sphere-packing upper bound on channel capacity. The derived upper bound is improved over the previous spherepacking upper bounds in [8], [9] but not over the upper bound in [3]. The upper bound in [3] has the advantage over the sphere-packing upper bounds that no optimization is required in calculation. However, our upper bound has a simpler expression.

\section{Geometrical Model of the Upper Bound on OPTICAL CHANNEL CAPACITY}

The $m$ independent transmitted symbols are denoted as $\mathbf{x}=\left(x_{1}, x_{2}, \cdots, x_{m}\right)$. Considering the constraints of the nonnegativity of the signal and the average optical power $P$ in VLC channel, the admissible set of the transmitted symbols is defined as

$$
\mathscr{A}(P)=\left\{\mathbf{x} \in \mathbb{R}^{m+}: \frac{1}{m}\|\mathbf{x}\|_{1} \leq P\right\},
$$

where $\|\cdot\|_{1}$ denotes $\ell_{1}$ norm. These elements in the admissible set would form a regualr $m$-simplex [11], and the received signal is expressed as

$$
\mathbf{y}=h \mathbf{x}+\mathbf{n},
$$

where $h$ is the channel gain between the transmitter and receiver.n is the noise vector and its elements follow the Gaussian distribution with zero mean and the variance of $\sigma^{2}$.

According to [7], from the geometrical point of view, each element in the signal vector $h \mathbf{x}$ is surrounded by a small uncertainty religion caused by the noise. When the noise is white and Gaussian, the perturbations of the received samples are independent. If $m$ is large enough, the perturbation would lie within a $m$-dimensional sphere $\mathscr{B}_{m}(\rho)$ with the radius 
$\rho$ centered at the original signal point. The volume of $m$ dimensional sphere $\mathscr{B}_{m}(\rho)$ is expressed as [7]

$$
V\left(\mathscr{B}_{m}(\rho)\right)=\frac{\pi^{m / 2}}{\Gamma\left(\frac{m}{2}+1\right)} \rho^{m}
$$

where $\Gamma(\cdot)$ is Gamma Function and $\rho=\sqrt{m} \sigma$.

In another word, the received signal would fall into the set $\mathscr{O}(P, \rho)$ defined as the outer parallel body to the admissible set $\mathscr{A}(P)$ at distance $\rho$ with the probability near to one, which is expressed as

$\mathscr{O}(P, \rho)=\left\{\mathbf{y} \in \mathbb{R}^{m}: \mathbf{y}=h \mathbf{x}+\mathbf{n}, \mathbf{x} \in \mathscr{A}(P), \mathbf{n} \in \mathscr{B}_{m}(\rho)\right\}$

Then, the number of nonoverlapping spheres packed in the outer parallel body $\mathscr{O}(P, \rho)$ to the admissible set $\mathscr{A}(P)$ is the maximum number of the different transmitted symbols to be distinguishable and can be defined as the upper bound on channel capacity, which is given by [7]

$$
C \leq \lim _{m \rightarrow \infty} \frac{1}{m} \log _{2} \frac{V(\mathscr{O}(P, \rho))}{V\left(\mathscr{B}_{m}(\rho)\right)}
$$

\section{DERIVATION OF THE UPPER BOUND ON OPTICAL ChANNEL CAPACITY}

In terms of the intrinsic volumes, the volume of the outer parallel body $V(\mathscr{O}(P, \rho))$ is expressed as [12]

$$
V(\mathscr{O}(P, \rho))=\sum_{k=0}^{m} V_{k}(P) V\left(\mathscr{B}_{m-k}(\rho)\right),
$$

where the intrinsic volumes $V_{k}(P)$ is given by

$$
V_{k}(P)=\eta_{k}(m h P)^{k}, \quad k=0,1, \cdots, m,
$$

and

$$
\eta_{k}= \begin{cases}\frac{1}{m !}, & \text { if } k=m ; \\
\left(\begin{array}{c}
m \\
k
\end{array}\right) \frac{1}{k ! 2^{m-k}}+\left(\begin{array}{c}
m \\
k+1
\end{array}\right) \frac{\sqrt{k+1}}{k ! \sqrt{\pi}^{m-k}} & \\
\times \int_{0}^{+\infty} e^{-x^{2}}\left(\int_{-\infty}^{x / \sqrt{k+1}} e^{-y^{2}} d y\right)^{m-k-1} d x, & \text { if } 0 \leq k<m .\end{cases}
$$

Substituting (3), (6) and (7) into (5), the upper bound can be rewritten as

$$
\begin{aligned}
C & \leq \lim _{m \rightarrow \infty} \frac{1}{m} \log _{2} \sum_{k=0}^{m} \frac{\Gamma\left(\frac{m}{2}+1\right)(m h P)^{k} \eta_{k}}{\Gamma\left(\frac{m-k}{2}+1\right) \pi^{\frac{k}{2}} \rho^{k}} \\
& \leq \lim _{m \rightarrow \infty} \frac{1}{m} \log _{2}\left[(m+1) \sup _{k \in \chi} \frac{\Gamma\left(\frac{m}{2}+1\right)(m h P)^{k} \eta_{k}}{\Gamma\left(\frac{m-k}{2}+1\right) \pi^{\frac{k}{2}} \rho^{k}}\right] \\
& =\lim _{m \rightarrow \infty} \frac{1}{m} \log _{2} \sup _{k \in \chi} \frac{\Gamma\left(\frac{m}{2}+1\right)(m h P)^{k} \eta_{k}}{\Gamma\left(\frac{m-k}{2}+1\right) \pi^{\frac{k}{2}} \rho^{k}},
\end{aligned}
$$

where $\chi=\{0,1, \cdots, m\}$.

To derive a simple closed-form upper bound, the coefficint $\eta_{k}$ is considered.

Lemma 1: For every $0 \leq k<m$, there is an inequality for $\eta_{k}$, such that

$$
\eta_{k}<\left(\begin{array}{c}
m+1 \\
k+1
\end{array}\right) \frac{k+1}{k !(m-k)\left(\frac{k}{\beta}+1\right)} \alpha^{-\frac{k}{\beta}} \frac{2^{m-k}-1}{2^{m-k}},
$$

where $\alpha$ and $\beta$ are positive real coefficients of the tight lower bound (i.e. $\alpha \exp \left(-\beta x^{2}\right)$ ) on the complementary error function $\operatorname{erfc}(\mathrm{x})$.

Proof: See Appendix A.

$$
\begin{aligned}
& C \leq \lim _{m \rightarrow \infty} \frac{1}{m} \log _{2} \sup _{k \in \chi} \frac{\Gamma\left(\frac{m}{2}+1\right)(m h P)^{k} \eta_{k}}{\Gamma\left(\frac{m-k}{2}+1\right) \pi^{\frac{k}{2}} \rho^{k}} \\
& <\lim _{m \rightarrow \infty} \frac{1}{m} \log _{2} \sup _{k \in \chi}\left\{\frac{\Gamma\left(\frac{m}{2}+1\right) \Gamma(m+2)}{[\Gamma(k+1)]^{2} \Gamma\left(\frac{m-k}{2}+1\right) \Gamma(m-k+1)} \frac{1}{(m-k)} \frac{m^{\frac{k}{2}}}{\frac{k}{\beta}+1}\left(\frac{h P}{\sigma}\right)^{k} \frac{2^{m-k}-1}{2^{m-k}} \pi^{-\frac{k}{2}} \alpha^{-\frac{k}{\beta}}\right\} \\
& \leq \lim _{m \rightarrow \infty} \frac{1}{m} \log _{2} \sup _{c \in \zeta}\left\{\frac{\Gamma\left(\frac{m}{2}+1\right) \Gamma(m+2)}{[\Gamma(c m+1)]^{2} \Gamma\left(\frac{m-c m}{2}+1\right) \Gamma(m-c m+1)} \frac{1}{(m-c m)} \frac{m^{\frac{c m}{2}}}{\frac{c m}{\beta}+1}\left(\frac{h P}{\sigma}\right)^{c m} \frac{2^{m-c m}-1}{2^{m-c m}} \pi^{-\frac{c m}{2}} \alpha^{-\frac{c m}{\beta}}\right\} \\
& =\sup _{c \in \zeta}\{\underbrace{\lim _{m \rightarrow \infty} \frac{1}{m} \log _{2}\left\{\frac{\Gamma\left(\frac{m}{2}+1\right) \Gamma(m+2)}{[\Gamma(c m+1)]^{2} \Gamma\left(\frac{m-c m}{2}+1\right) \Gamma(m-c m+1)} \frac{1}{(m-c m)} \frac{m^{\frac{c m}{2}}}{\frac{c m}{\beta}+1}\right\}}_{\sigma_{c m}} \\
& \underbrace{\lim _{m \rightarrow \infty} \log _{2}\left(1-\frac{1}{2^{m-c m}}\right)^{\frac{1}{m}}}_{\delta_{c m}}+\lim _{m \rightarrow \infty} \frac{1}{m} \log _{2} \pi^{-\frac{c m}{2}} \alpha^{-\frac{c m}{\beta}}\left(\frac{h P}{\sigma}\right)^{c m}\} \\
& \leq \sup _{c \in \zeta} \underbrace{\log _{2}\left[\frac{\left(\frac{e}{2 \pi}\right)^{\frac{c^{*}}{2}} \alpha^{-\frac{c^{*}}{\beta}}}{\left(c^{*}\right)^{2 c^{*}}\left(1-c^{*}\right)^{\frac{3}{2}\left(1-c^{*}\right)}}\left(\frac{h P}{\sigma}\right)^{c^{*}}\right]}_{w(c)} .
\end{aligned}
$$


Based on Corollary 1 in Appendix B, a useful choice for $\alpha$ and $\beta$ are 0.74 and 1.75. Then, according to Lemma 1, the new upper bound is given in the following theorem.

Theorem 1: The channel capacity is upper-bounded by

$$
C<\log _{2}\left[\frac{\left(\frac{e}{2 \pi}\right)^{\frac{c^{*}}{2}} \alpha^{-\frac{c^{*}}{\beta}}}{\left(c^{*}\right)^{2 c^{*}}\left(1-c^{*}\right)^{\frac{3}{2}\left(1-c^{*}\right)}}\left(\frac{h P}{\sigma}\right)^{c^{*}}\right] .
$$

where $c^{*} \in[0,1]$ is obtained by

$$
\begin{aligned}
& 2 \log _{2} c^{*}-\frac{3}{2} \log _{2}\left(1-c^{*}\right) \\
& =-\frac{1}{\ln 4}+\frac{1}{2} \log _{2}\left(\frac{e}{2 \pi}\right)-\frac{1}{\beta} \log _{2} \alpha+\log _{2}\left(\frac{h P}{\sigma}\right) .
\end{aligned}
$$

Proof: According to (9) and (10), the upper bound can be obtained by the inequality (13), where we define $\zeta=\{c \in$ $\mathbb{R} \mid 0 \leq c \leq 1\}$ and the value of $\sigma_{c m}$ and $\delta_{c m}$ are calculated in Appendix $\mathrm{C}$ and $\mathrm{D}$, respectively.

Furthermore, the existence of the supremum of $w(c)$ in (13) is investigated. As the second order derivation of $w(c)$ is non-positive, $w(c)$ is a concave function. Thus, for $c \in \zeta$, a maximum value exists. An optimal $c$ denoted as $c^{*}$ is then obtained by the equation $w^{\prime}\left(c^{*}\right)=0$, which is defined as (12).

It should be pointed out that our upper bound and the previous sphere-packing upper bounds in [8], [9] are all derived using the Steiner-Minkowski formula. The difference lies in the approximation method for the intrinsic volumes of the simplex, which eventually leads to the different performance in some OSNR region.

\section{NumericAl Results}

In this section, the numerical results about the upper bound on channel capacity under different optical signal-to-noise ratio $(\mathrm{OSNR}=P / \sigma)$ are discussed. The channel gain $h$ is set to be 1 . The optimal value of $c^{*}$ is numerically calculated based on (12), which is substituted in (11) to achieve the upper bound on channel capacity.

To verify the tightness of the proposed upper bound derived in this letter, we compared it with the upper bounds in [3], [8], [9] and the lower bounds in [3], [9]. As illustrated in Fig. 1, our proposed upper bound are tighter both at low and high OSNRs than the upper bound in [8]. Although our bound is looser at low OSNRs compared to the upper bound in [9], it is tighter at high OSNRs. Since typical illumination levels offer high OSNRs in visible light communications [8], this improvement in high OSNR region is significant. In addition, the upper bound in [3] is presented in Fig. 1. It can be observed that the upper bound in [3] is tighter than our upper bound at overall OSNRs. However, our upper bound has a simpler expression.

On the other hand, our new upper bound is also compared with the lower bounds in [3], [9]. It can be seen that there exists a gap between our bound and the lower bounds in [3], [9] at low OSNRs. However, the gap is narrowed as 0.25 bits/channel use in high OSNR region.

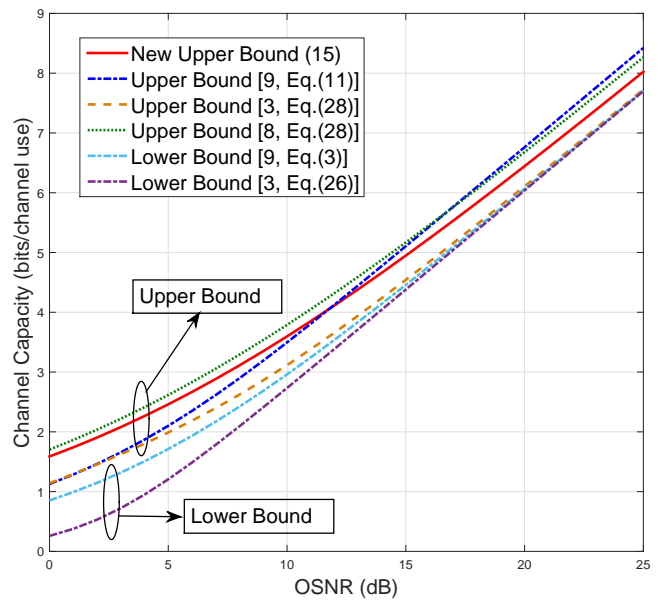

Fig. 1. Capacity bound comparison for the optical wireless channel.

\section{APPENDIX A}

PROOF OF THEOREM 1

Firstly, we defined an intermediate variable $\mu_{k}$ as follows

$$
\begin{aligned}
& \mu_{k}= \frac{\sqrt{k+1}}{\sqrt{\pi}} \int_{0}^{+\infty} e^{-x^{2}}\left(\int_{-\infty}^{x / \sqrt{k+1}} e^{-y^{2}} d y\right)^{m-k-1} d x \\
& \underline{t=\frac{x}{\sqrt{k+1}}} \frac{k+1}{\sqrt{\pi}} \int_{0}^{+\infty} e^{-(1+k) t^{2}}\left[1-\frac{1}{2} \operatorname{erfc}(t)\right]^{m-k-1} d t \\
& \leq \frac{k+1}{\sqrt{\pi}} \int_{0}^{+\infty} e^{-t^{2}}\left[\frac{1}{\alpha} \operatorname{erfc}(t)\right]^{\frac{k}{\beta}}\left[1-\frac{1}{2} \operatorname{erfc}(t)\right]^{m-k-1} d t \\
& \underline{\underline{=} \frac{1}{2} \operatorname{erfc}(t)}(k+1)\left(\frac{2}{\alpha}\right)^{\frac{k}{\beta}} \int_{0}^{\frac{1}{2}} \tau^{\frac{k}{\beta}}(1-\tau)^{m-k-1} d \tau \\
&=\frac{k+1}{2^{m-k}} \alpha^{-\frac{k}{\beta}}\left[\frac{1}{\frac{k}{\beta}+1}+\frac{m-k-1}{\left(\frac{k}{\beta}+1\right)\left(\frac{k}{\beta}+2\right)}\right. \\
&\left.\quad+\cdots+\frac{(m-k-1) !}{\left(\frac{k}{\beta}+1\right)\left(\frac{k}{\beta}+2\right) \cdots\left(\frac{k}{\beta}+m-k\right)}\right] \\
&<\frac{k+1}{\frac{k}{\beta}+1} \frac{1}{2^{m-k}} \alpha^{-\frac{k}{\beta}} \\
& \quad \times\left[1+\frac{m-k-1}{1 \times 2}+\cdots+\frac{(m-k-1) !}{(m-k) !}\right] \\
&=\frac{k+1}{\frac{k}{\beta}+1} \frac{1}{2^{m-k}} \alpha^{-\frac{k}{\beta}} \sum_{i=0}^{m-k-1} \frac{C_{m-k-1}^{i}}{i+1} \\
&=\frac{k+1}{(m-k)\left(\frac{k}{\beta}+1\right)} \alpha^{-\frac{k}{\beta}} \frac{2^{m-k}-1}{2^{m-k}},
\end{aligned}
$$

where the first inequality is satisfied if the lower bound on $\operatorname{erfc}(\mathrm{x})$ is applied (See Corollary 1, Appendix B) and we also have

$$
\begin{aligned}
\mu_{k} & =\frac{k+1}{\sqrt{\pi}} \int_{0}^{+\infty} e^{-(1+k) t^{2}}\left[1-\frac{1}{2} \operatorname{erfc}(t)\right]^{m-k-1} d t \\
& >\frac{k+1}{\sqrt{\pi}} \int_{0}^{+\infty} e^{-(1+k) t^{2}}\left(\frac{1}{2}\right)^{m-k-1} d t>\frac{1}{2^{m-k}},
\end{aligned}
$$

where the inequality is satisfied because erfc(x) is not exceeding 1. 
Thus, substituting (13) and (14) into (8), $\eta_{k}$ can be rewritten as

$$
\begin{aligned}
\eta_{k} & =\left(\begin{array}{c}
m \\
k
\end{array}\right) \frac{1}{k ! 2^{m-k}}+\left(\begin{array}{c}
m \\
k+1
\end{array}\right) \frac{1}{k !} \mu_{k} \\
& <\left(\begin{array}{c}
m+1 \\
k+1
\end{array}\right) \frac{\mu_{k}}{k !} \\
& <\left(\begin{array}{c}
m+1 \\
k+1
\end{array}\right) \frac{k+1}{k !(m-k)\left(\frac{k}{\beta}+1\right)} \alpha^{-\frac{k}{\beta}} \frac{2^{m-k}-1}{2^{m-k}} .
\end{aligned}
$$

\section{APPENDIX B}

\section{EXPONENTIAL-TYPE LOWER BOUND ON erfc(x)}

The exponential-type bound on the complementary error function $\operatorname{erfc}(\mathrm{x})$ is given by

$$
L(x)=\alpha \exp \left(-\beta x^{2}\right)
$$

where $\alpha$ and $\beta$ are positive and real numbers. To analyze the lower bound on $\operatorname{erfc}(\mathrm{x})$, we define the error function $g(x)$, which satisfies

$$
g(x)=\operatorname{erfc}(\mathrm{x})-\alpha \exp \left(-\beta x^{2}\right) .
$$

Lemma 2: For $x \in(0,+\infty), g(x)$ has a minimum point if

$$
\sqrt{\frac{2 e}{\pi}} \frac{\sqrt{\beta-1}}{\beta}<\alpha<1 \text { and } \beta>1 .
$$

Proof: From (17), we have $g^{\prime}(x)=\exp \left(-x^{2}\right)\left(-\frac{2}{\sqrt{\pi}}+\right.$ $\left.2 \alpha \beta x \exp \left((1-\beta) x^{2}\right)\right)$. To analyze the characteristics of $g(x)$, we first consider the function $f(x)$, which is expressed as

$$
f(x)=-\frac{2}{\sqrt{\pi}}+2 \alpha \beta x \exp \left((1-\beta) x^{2}\right) .
$$

Then, we have $f^{\prime}(x)=2 \alpha \beta\left(2(1-\beta) x^{2}+1\right) \exp ((1-$ $\left.\beta) x^{2}\right)$. Thus, if $\beta>1, f(x)$ has an extreme point when $x_{0}=\frac{1}{\sqrt{2(\beta-1)}}$. Further, we have $f^{\prime \prime}\left(x_{0}\right)<0$. Therefore, $f\left(x_{0}\right)=-\frac{2}{\sqrt{\pi}}+\frac{\sqrt{2} \alpha \beta}{\sqrt{\beta-1}} \exp \left(-\frac{1}{2}\right)$ is a maximum value. If $\alpha>$ $\sqrt{\frac{2 e}{\pi}} \frac{\sqrt{\beta-1}}{\beta}$ is satisfied, we have $f\left(x_{0}\right)>0$. Since $f(0)<0$ and $f(\infty)<0, g(x)$ is monotonically decreasing at first, then monotonically increasing and monotonically decreasing in the end. Thus, $g(x)$ has a minimum point.

Theorem 2: $L(x)$ is a lower bound on $\operatorname{erfc}(\mathrm{x})$ if

$$
\sqrt{\frac{2 e}{\pi}} \frac{\sqrt{\beta-1}}{\beta}<\alpha<1, \quad \beta>1 \quad \text { and } \quad g\left(x_{1}\right) \geqslant 0
$$

where $x_{1}$ is the smallest root of $f(x)$ defined in (19).

Proof: If $\sqrt{\frac{2 e}{\pi}} \frac{\sqrt{\beta-1}}{\beta}<\alpha<1$ and $\beta>1$, we have $f(0)<$ $0, f\left(x_{0}\right)>0$, and $f(+\infty)<0$. Thus, $f(x)$ has no less than two roots and the smallest root $x_{1}$ determines that $g(x)$ has a minimum point. In addition, we have $g(0)=1-\alpha>0$ and $g(\infty)=0$. Considering the monotonicity of $g(x)$, if $g\left(x_{1}\right) \geq 0$ is satisfied, $L(x)$ is a lower bound on $\operatorname{erfc}(\mathrm{x})$.

Corollary 1: An optimal $L^{*}(x)$ is obtained with the optimization problem to minimize the mean squared error between $\operatorname{erfc}(\mathrm{x})$ and $L(x)$ when $x \in[a, b]$, which is formulated as

$$
\begin{aligned}
& \min _{\alpha, \beta} \frac{1}{b-a} \int_{a}^{b} g(x)^{2} d x \\
& \text { s.t. } \sqrt{\frac{2 e}{\pi}} \frac{\sqrt{\beta-1}}{\beta}<\alpha<1, \quad \beta>1, \quad g\left(x_{1}\right) \geqslant 0,
\end{aligned}
$$

where $x_{1}$ is the smallest root of $f(x)$ defined in (19).

Proof: From Theorem 2, with the metric of mean squared error, a tight lower bound on $\operatorname{erfc}(\mathrm{x})$ can be obtained.

\section{APPENDIX C}

THE VALUE OF $\sigma_{m s}$

Using the Stirling's inequality, which is given by [13]

$$
\sqrt{2 \pi n}\left(\frac{n}{e}\right)^{n} e^{\frac{1}{12 n+1}}<\Gamma(n+1)<\sqrt{2 \pi n}\left(\frac{n}{e}\right)^{n} e^{\frac{1}{12 n}},
$$

and based on the squeeze theorem, we have

$$
\begin{gathered}
\sigma_{m s}=\log _{2}\left[\frac{\left(\frac{e}{2}\right)^{\frac{c}{2}}}{c^{2 c}(1-c)^{\frac{3}{2}(1-c)}}\right] . \\
\text { APPENDIX D } \\
\text { THE VALUE OF } \delta_{m s}
\end{gathered}
$$

For $0 \leq x \leq 1$, we have

$$
\lim _{n \rightarrow+\infty}\left(1-x^{n}\right)^{\frac{1}{n}}=1 .
$$

After that, we get

$$
\delta_{m s}=\lim _{m \rightarrow \infty} \log _{2}\left(1-\frac{1}{2^{m-c m}}\right)^{\frac{1}{m}}=0 .
$$

\section{REFERENCES}

[1] R. Zhang, J. Wang, Z. Wang, Z. Xu, C. Zhao, and L. Hanzo, "Visible light communications in heterogeneous networks: paving the way for user-centric design," IEEE Wireless Commun., vol. 22, no. 2, pp. 8-16, Apr. 2015.

[2] Q. Wang, Z. Wang, L. Dai, "Asymmetrical hybrid optical OFDM for visible light communications with dimming control," IEEE Photon. Technol. Lett., vol. 27, no. 9, pp. 974-977, May 2015.

[3] A. Lapidoth, S. M. Moser, M. A. Wigger, "On the capacity of free-space optical intensity channels," IEEE Trans. Inf. Theory, vol. 55, no. 10, pp. 4449-4461, Oct. 2009.

[4] A. Nuwanpriya, S. Ho, and C. S. Chen, "Indoor MIMO visible light communications: novel angle diversity receivers for mobile users," IEEE J. Sel. Areas Commun., vol. 33, no. 9, pp. 1780-1792, Sept. 2015.

[5] S. Dimitrov and H. Hass, "Information rate of OFDM-based optical wireless communication systems with nonlinear distortion," J. Lightwave Technol., vol. 31, no. 6, pp. 918-929, Mar. 2013.

[6] J. M. Kahn and J. R. Barry, "Wireless infrared communications," Proc. IEEE, vol. 85, no. 2, pp. 265-298, Feb. 1997.

[7] C. E. Shannon, "Communication in the presence of noise," Proc. IRE, vol. 37, pp. 10-21, Jan. 1949.

[8] J. Wang, Q. Hu, J. Wang, M. Chen, and J. Wang, "Tight bounds on channel capacity for dimmable visible light communications," $J$. Lightwave Technol., vol. 31, no. 23, pp. 3771-3779, Dec. 2013.

[9] A. Farid and S. Hranilovic, "Capacity bounds for wireless optical intensity channels with Gaussian noise," IEEE Trans. Inf. Theory, vol. 56, no. 12 , pp. 6066-6077, Dec. 2010.

[10] A. Chaaban, J. Morvan, M. Alouini, "Free-space optical communications: capacity bounds, approximations, and a new spherepacking perspective," Technical Report, May 2015, Available at: http://repository.kaust.edu.sa/kaust/handle/10754/552096.

[11] W. Rudin, Principle of Mathematical Analysis. New York: McGraw-Hill, 1976.

[12] U. Betke and M. Henk, "Intrinsic volumes and lattice points of crosspolytope," Monatsh. Mathematik, vol. 115, no. 1-2, pp. 27-33, 1993.

[13] H. Robbins, "A remark on Stirling's formula," Amer. Math. Mon., vol. 62, no. 1, pp. 26-29, 1955. 